\title{
Reduced graphene oxide and graphene composite materials for improved gas sensing at low temperature $\uparrow$
}

\author{
Alexander Zöpfl, ${ }^{a}$ Michael-Maximilian Lemberger, ${ }^{a}$ Matthias König, ${ }^{b}$ \\ Guenther Ruhl, ${ }^{b}$ Frank-Michael Matysik ${ }^{a}$ and Thomas Hirsch*a
}

Received 30th April 2014, Accepted 27th June 2014

DOI: $10.1039 / c 4 f d 00086 b$

Reduced graphene oxide ( $\mathrm{rGO}$ ) was investigated as a material for use in chemiresistive gas sensors. The carbon nanomaterial was transferred onto a silicon wafer with interdigital gold electrodes. Spin coating turned out to be the most reliable transfer technique, resulting in consistent $\mathrm{rGO}$ layers of reproducible quality. Fast changes in the electrical resistance at a low operating temperature of $85^{\circ} \mathrm{C}$ could be detected for the gases $\mathrm{NO}_{2}, \mathrm{CH}_{4}$ and $\mathrm{H}_{2}$. Especially upon adsorption of $\mathrm{NO}_{2}$ the high signal changes allowed a minimum detection of $0.3 \mathrm{ppm}(S / N=3)$. To overcome the poor selectivity, rGO was chemically functionalized with octadecylamine, or modified by doping with metal nanoparticles such as $\mathrm{Pd}$ and $\mathrm{Pt}$, and also metal oxides such as $\mathrm{MnO}_{2}$, and $\mathrm{TiO}_{2}$. The different response patterns for six different materials allowed the discrimination of all of the test gases by pattern recognition based on principal component analysis.

\section{Introduction}

The simple and reliable monitoring of gas concentrations is important in everyday-life. In industrial processes hazardous gases need to be controlled to guarantee safety. Controlling air quality can save energy in automated air conditioning. Also the detection of environmental pollution (like $\mathrm{NO}_{x}$ ) is of great interest. ${ }^{1}$ Up to now, solid-state gas sensors based on metal oxide chemiresistors are well established and widely used in detecting gases. ${ }^{2,3}$ They are operated at high temperatures, ${ }^{4}$ which consumes excessive energy and limits their long-term stability, thus leading to the development of new gas sensor concepts which overcome these drawbacks. In the last decade carbon nanomaterials, like carbon nanotubes ${ }^{5,6}$ or graphene, ${ }^{7-10}$ were shown to be capable of gas detection due to

${ }^{a}$ Institute of Analytical Chemistry, Chemo- and Biosensors, University of Regensburg, Universitaetsstrasse 31, 93053 Regensburg, Germany. E-mail: alexander.zoepfl@ur.de; michael.lemberger@ur.de; frank-michael. matysik@ur.de; thomas.hirsch@ur.de

${ }^{b}$ Infineon Technologies AG, 93049 Regensburg, Germany. E-mail: Guenther.Ruhl@infineon.com; Matthias. Koenig@infineon.com

$\dagger$ Electronic supplementary information (ESI) available. See DOI: 10.1039/c4fd00086b 
their high sensitivity to various gases even at low operating temperatures. Especially graphene has recently attracted intense attention because of its high charge carrier mobility (15 $000 \mathrm{~cm}^{2} \mathrm{~V}^{-1} \mathrm{~s}^{-1}$ under ambient conditions) and large surface area as a consequence of having a thickness of only one atom and the absence of any bulk phase. .112 $^{12}$

Many different preparation methods for graphene are known so far, but only a few of them are applicable in terms of sensor preparation. Chemically derived graphene obtained by the reduction of graphene oxide $(\mathrm{GO})^{\mathbf{1 3 , 1 4}}$ is inexpensive and the synthesis is easily scalable. Due to a more defective structure its electrical properties are not as outstanding as those of pristine graphene, ${ }^{15,16}$ but are still suitable for sensitive gas detection. ${ }^{17}$ It has also been described that graphene with a more defective structure shows an improved adsorption of gas molecules. ${ }^{18}$ Furthermore, reduced graphene oxide (rGO) can be dispersed in solutions, simplifying the transfer to a sensor set-up by e.g. spraying, printing and casting methods. ${ }^{19,20}$

Similar to solid state gas sensors, gas adsorption on graphene leads to a change in its electrical resistance (Fig. 1). Therefore, any kind of interaction between graphene sheets and adsorbates influences the electronic structure of the graphene, leading to an altered charge carrier concentration or electrical conductance of the material, respectively. ${ }^{17,21}$ Nevertheless, up to now most of such sensor concepts have been demonstrated only in inert gas mixtures or at elevated temperatures. For any practical application it is necessary to overcome the limitations of poor selectivity. ${ }^{7}$ Many works address this issue by chemically modifying the carbon material with functional groups or doping with metals and metal oxides. Graphene composite materials have been used in many different sensing applications and can be easily produced either by simply mixing two different materials together or by in situ nanoparticle growth on graphene in a suspension. $^{22-24}$

Here we report on rGO as a sensitive material for gas detection and the possibilities of functionalization to introduce selectivity. Reduced graphene oxide

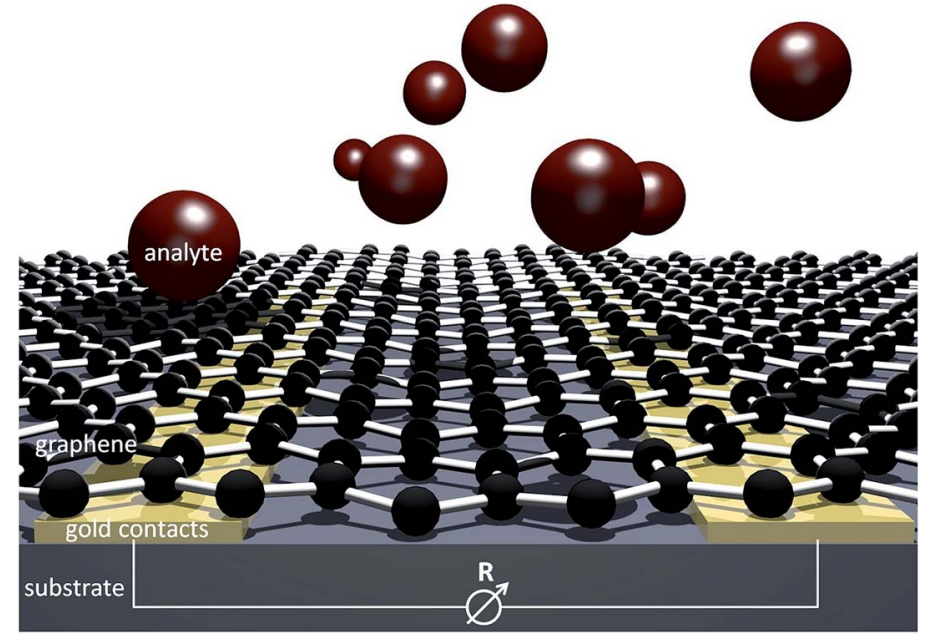

Fig. 1 Scheme of the principle of chemiresistor gas sensors based on graphene. 
was prepared by the chemical reduction of GO, which was obtained from the oxidation of graphite. The resulting product can be dispersed in water, enabling an easy transfer to pre-structured microelectrodes comprising an interdigital structure. Application of rGO via spin coating was optimized and resulted in consistent layers of reproducible quality in terms of the electrical properties. The conductance of such modified electrodes was measured in the presence of various gases diluted in synthetic air $\left(\mathrm{NO}_{2}, \mathrm{CH}_{4}\right.$, and $\left.\mathrm{H}_{2}\right)$ at moderate temperatures $\left(85{ }^{\circ} \mathrm{C}\right)$. Chemical modifications were applied by the insertion of functional groups and by doping with metals and metal oxides. The resulting materials were characterized and tested for their gas sensing behaviour. It was demonstrated that a combination of simple modifications of rGO can lead to sensors with different characteristics, allowing a pattern detection of various gases.

\section{Experimental}

\subsection{Materials and instrumentation}

Unless otherwise stated, all chemicals were purchased from Merck (Darmstadt, Germany) or Sigma-Aldrich (Steinheim, Germany) and used without further purification. Ultra-pure water $\left(0.055 \mu \mathrm{S} \mathrm{cm}^{-1}\right)$ was used in all experiments. All gases and mixtures were purchased from Linde AG.

Raman spectra were recorded on a Thermo Scientific DXR Raman microscope with a $532 \mathrm{~nm}$ excitation laser $(10 \mathrm{~mW})$. Scanning electron microscopy (SEM) was performed with a Zeiss Ultra 55, EDX on a JOEL JSM-6700F and TGA-FTIR with a Netzsch Iris TG209 connected to a Bruker Equinox 55, all at Infineon Technologies AG Regensburg. Transmission electron microscopy (TEM) was performed using a $120 \mathrm{kV}$ Philips CM12 microscope.

\subsection{Synthesis of reduced graphene oxide and modifications}

GO was synthesized using a slightly modified Hummers method. ${ }^{13}$ Briefly, $100 \mathrm{mg}$ graphite (China flake graphite, K. W. Thielmann \& Cie KG) was mixed with $75 \mathrm{mg}$ $\mathrm{NaNO}_{3}, 7.5 \mathrm{~mL}$ conc. $\mathrm{H}_{2} \mathrm{SO}_{4}$ and $450 \mathrm{mg} \mathrm{KMnO}_{4}$. The mixture was sonicated for $3 \mathrm{~h}$ and stirred for 3 days at room temperature. Subsequently, $7.5 \mathrm{~mL}$ of $5 \% \mathrm{H}_{2} \mathrm{SO}_{4}$ was added and the mixture was stirred under reflux for $2 \mathrm{~h}$ at $100{ }^{\circ} \mathrm{C}$, followed by the addition of $1.5 \mathrm{~mL}$ of $30 \% \mathrm{H}_{2} \mathrm{O}_{2}$ under constant stirring for $1 \mathrm{~h}$ at room temperature. For purification the obtained GO was washed with the following solutions: four times in $3 \% \mathrm{H}_{2} \mathrm{SO}_{4}$ with $0.5 \% \mathrm{H}_{2} \mathrm{O}_{2}$; two times in $3 \% \mathrm{HCl}$; and three times in water. The product was dialyzed against water (14 kDa cut-off) for 10 days.

To obtain rGO, ${ }^{14} 7 \mathrm{~mL}$ of a GO suspension $\left(0.5 \mathrm{mg} \mathrm{mL}^{-1}\right)$ was mixed with $31 \mu \mathrm{L}$ $\mathrm{NH}_{3}$ (32\% in water). After adding $5 \mu \mathrm{L}$ of $98 \%$ hydrazine hydrate the reaction mixture was refluxed for $1 \mathrm{~h}$ at $100{ }^{\circ} \mathrm{C}$. Finally the resulting black suspension was washed with water.

\section{Modification with octadecylamine}

To modify rGO with octadecylamine (ODA), the synthesis of surface functionalized graphene by Wang et al. was adapted. ${ }^{25} 5 \mathrm{mg}$ GO was suspended in $5 \mathrm{~mL}$ dichlorobenzene. After adding $50 \mathrm{mg}$ ODA, the reaction mixture was sonicated for $24 \mathrm{~h}$ at $80^{\circ} \mathrm{C}$. To get rid of excess ODA and dichlorobenzene, the reaction mixture 
was treated with $40 \mathrm{~mL}$ ethanol resulting in a black precipitate. The mixture was centrifuged and the resulting solid was washed twice with ethanol and toluene. This washing step was repeated once more and the product (rGO-ODA) was dried in air.

\section{Modification with $\mathrm{MnO}_{2}$}

A synthesis for GO- $\mathrm{MnO}_{2}$ nano composites was adapted. ${ }^{26} 5.1 \mathrm{mg}$ GO and $21 \mathrm{mg}$ $\mathrm{MnCl}_{2} \cdot 4 \mathrm{H}_{2} \mathrm{O}$ were dispersed in $5 \mathrm{~mL}$ isopropanol and sonicated for $30 \mathrm{~min}$. The reaction mixture was refluxed at $83{ }^{\circ} \mathrm{C}$ under vigorous stirring. Afterwards a solution of $12 \mathrm{mg} \mathrm{KMnO}_{4}$ in $0.4 \mathrm{~mL}$ water was added and the slurry was refluxed at $83{ }^{\circ} \mathrm{C}$ for another $30 \mathrm{~min}$ before it was cooled to room temperature. The nano composite was then centrifuged and washed twice with water. The resulting brown product $\left(\mathrm{rGO}-\mathrm{MnO}_{2}\right)$ was dried at $60{ }^{\circ} \mathrm{C}$ overnight.

\section{Modification with $\mathrm{TiO}_{2}$}

For the functionalization of carbon nanomaterials $\mathrm{Li}$ et al. have reported the modification of single-walled carbon nanotubes with $\mathrm{TiO}_{2} \cdot{ }^{27}$ In an analogous way rGO was modified with $\mathrm{TiO}_{2}\left(\mathrm{rGO}^{-\mathrm{TiO}_{2}}\right): 1 \mathrm{mg}$ of $\mathrm{TiO}_{2}$ with an average particle size of $1 \mu \mathrm{m}$ was ground thoroughly. Subsequently the powder was dispersed in $2 \mathrm{~mL}$ water, sonicated for $1 \mathrm{~h}$ and $1 \mathrm{~mL}$ of an aqueous rGO suspension $\left(0.5 \mathrm{mg} \mathrm{mL}{ }^{-1}\right)$ was added. After another sonication for $1 \mathrm{~h}$ a grey suspension was formed where the modified rGO flakes tended to aggregate.

\section{Modification with Pd and Pt nanoparticles}

Both approaches were adapted from the preparation of graphene-metal nanocomposites by Xu et al. and modified slightly. ${ }^{28} 5 \mathrm{~mL}$ of a GO suspension $(1 \mathrm{mg}$ $\mathrm{mL}^{-1}$ ) was mixed with $10 \mathrm{~mL}$ ethylene glycol. Afterwards $0.5 \mathrm{~mL}$ of a $0.01 \mathrm{M}$ solution of a metal precursor $\left(\mathrm{K}_{2} \mathrm{PtCl}_{4}\right.$ or $\left.\mathrm{PdCl}_{2}\right)$ in water was added and the mixture was first stirred for $30 \mathrm{~min}$ at room temperature and then for $6 \mathrm{~h}$ at $100{ }^{\circ} \mathrm{C}$. During the heating in both cases the colour became black indicating that the GO had been reduced by ethylene glycol. The resulting suspensions containing the metal doped carbon nanomaterials (rGO-Pd and rGO-Pt) were centrifuged for $5 \mathrm{~min}$ at $3000 \mathrm{rpm}$ and washed three times with water.

\subsection{Electrode preparation}

Suspensions of rGO and the modifications were deposited on gold microelectrodes (Fig. 2) via spin coating. The respective suspensions in a $1: 1$-mixture of water and isopropanol $\left(0.25 \mathrm{mg} \mathrm{mL}^{-1}\right)$ were sonicated for $10 \mathrm{~min}$ before $2 \mu \mathrm{L}$ of each was spin coated onto the interdigital electrode structure with a Laurell WS400BZ-6NPP/LITE spin coater (5 s at $500 \mathrm{rpm}$ and $20 \mathrm{~s}$ at $3000 \mathrm{rpm}$ ). Afterwards the electrodes were heated to approximately $230{ }^{\circ} \mathrm{C}$ for $30 \mathrm{~s}$ on a hot plate.

\subsection{Gas measurements}

Gas measurements were performed on a homemade gas mixing device, consisting of mass flow controllers (UFC-8160A and UFC-1660 from Unit Instruments Inc.), a flow cell with temperature control by two heating elements, and a Pt-100 temperature sensor (Fig. SI $1 \dagger$ ). 


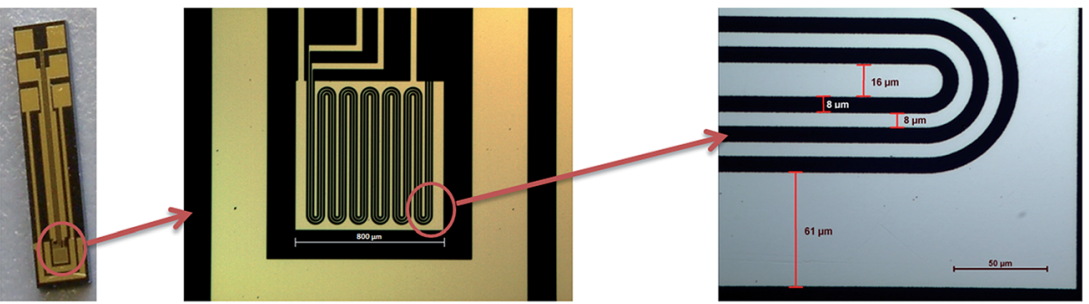

Fig. 2 Microelectrodes with an interdigital structure consisting of gold conducting paths on a $\mathrm{Si} / \mathrm{SiO}_{2}$ wafer substrate.

Measurement of the electrical resistance was accomplished by contacting the two gold conducting paths in the mid-position of the interdigital electrode structure (Fig. 2) using a Keithley 2400 sourcemeter at a constant bias voltage of $50 \mathrm{mV}$. Synthetic air $\left(\mathrm{N}_{2}: 80 \%, \mathrm{O}_{2}: 20 \%\right)$ was used as the carrier gas and was mixed with $300 \mathrm{ppm} \mathrm{NO}_{2}, 1 \% \mathrm{H}_{2}$ or $1 \% \mathrm{CH}_{4}$. The test gases were all diluted by the synthetic air. Furthermore the gas adsorption tests were accomplished at a constant gas flow of $100 \mathrm{sccm}(300 \mathrm{sccm}$ for the concentration dependency of $\mathrm{NO}_{2}$ ) and a constant temperature of $85{ }^{\circ} \mathrm{C}$. It was also necessary to heat the electrodes for $20 \mathrm{~s}$ at $230{ }^{\circ} \mathrm{C}$ before each measurement, ensuring that no analyte gas had been previously adsorbed by reaching the initial resistance. For measuring the influence of air humidity, the gas flow was piped through the head space of a flask containing water.

\section{Results and discussion}

\subsection{Choice of materials and sensor fabrication}

The transfer of graphene materials is still the most critical step in sensor preparation using this type of carbon nanomaterial. Graphene prepared by e.g. the CVD technique ${ }^{29}$ requires a sophisticated transfer method of the carbon nanomaterial from the metallic substrate to the substrate of choice. This can be done e.g. by a transfer with stamping methods, ${ }^{30}$ which leads to contamination of the graphene and therefore such processes lack reproducibility. In contrast, rGO suspensions can be easily processed. Reduced graphene is usually transferred to electrodes by drop casting, which results in layers of inhomogeneous thickness and low reproducibility. The sensitivity of chemoresistors strongly depends on the thickness of the conductive layer. Therefore we used an interdigital electrode structure which was covered with a thin layer of rGO via spin coating. Parameters affecting the spin coating, such as the concentration of rGO, the rotation speed and time, as well as the usage of certain additives like isopropanol for improving the spreading of the suspension were optimized to obtain consistent layers with reproducible total resistance.

\subsection{Characterization of the microelectrodes modified with reduced graphene oxide and composite materials}

Graphene oxide was prepared by a modified Hummers method and reduced with hydrazine as reported previously. ${ }^{\mathbf{1 4}}$ The size of the graphene flakes ranged from 
$100 \mathrm{~nm}$ to about $1 \mu \mathrm{m}$ as indicated by SEM studies (Fig. SI 2a †). The Raman spectrum of rGO (Fig. 3a) exhibits the typical broad D and G bands at $1342 \mathrm{~cm}^{-1}$ and $1602 \mathrm{~cm}^{-1}$. $^{31}$ The $\mathrm{D} / \mathrm{G}$ ratio and position of the $\mathrm{G}$ band indicates crystalline domains of several nanometers in size. ${ }^{32}$

Regarding the thermogravimetric analysis (TGA) of GO and rGO (Fig. 3b), a loss of mass can be observed at temperatures of about $200^{\circ} \mathrm{C}$. In FTIR-TGA (Fig. SI $3 \dagger$ ) this effect can be ascribed to a thermal reduction, where $\mathrm{CO}$ and $\mathrm{CO}_{2}$ are released. ${ }^{33}$ Graphene oxide loses about $25 \%$ of its mass, while rGO loses around $15 \%$, which shows that the reduction by hydrazine is incomplete.

Further, the rGO modified electrodes were heated to $230{ }^{\circ} \mathrm{C}$ to guarantee a thermal reduction. During this step the electrical resistance dropped from about $8.0 \pm 3.8 \mathrm{M} \Omega$ to $180 \pm 78 \mathrm{k} \Omega$. The oxidation process of graphite introduces many defects into the $\mathrm{sp}^{2}$ structure which act like a barrier for the electron flux, resulting in better conductance at higher levels of reduction. ${ }^{34}$

Functionalization of rGO was performed by wet chemical methods before the composite material was also transferred to the microelectrodes. Generally all dopants primarily bind/coordinate with the oxygen functionalities of rGO. Whereas ODA reacts most probably with the epoxy groups of $\mathrm{GO},{ }^{25}$ doping with metals and metal oxides usually includes the interaction of a precursor metal ion with the carboxyl groups of rGO. ${ }^{28,35}$ The successful modification of rGO was revealed by Raman studies. Peaks at 1125 and $1489 \mathrm{~cm}^{-1}$ (ODA), 140, 392, 510 and $644 \mathrm{~cm}^{-1}\left(\mathrm{TiO}_{2}\right)$, and 579 and $633 \mathrm{~cm}^{-1}\left(\mathrm{MnO}_{2}\right)$ were found (Fig. SI $\left.4 \dagger\right)$. Graphene composites with conductive Pt- and Pd-nanoparticles could be observed on distinct regions of rGO in SEM and TEM images. The average particle size was below $20 \mathrm{~nm}$ (Fig. SI 5 and SI $6 \dagger$ ). The elemental composition of the different modified materials was confirmed using energy-dispersive X-ray spectroscopy (EDS) (Fig. SI 7†).

Covalent attachment of functional groups ${ }^{36}$ or doping with metals and metal oxides $^{37}$ may change the electronic structure and level of $n$-/p-doping within the material, but may also increase the sensor surface or have a catalytic effect on gas adsorption.

\subsection{Gas sensor response}

To assess the effect of gas adsorption on the conductance of rGO, the electrical resistance of the coated microelectrodes was measured for different gases of
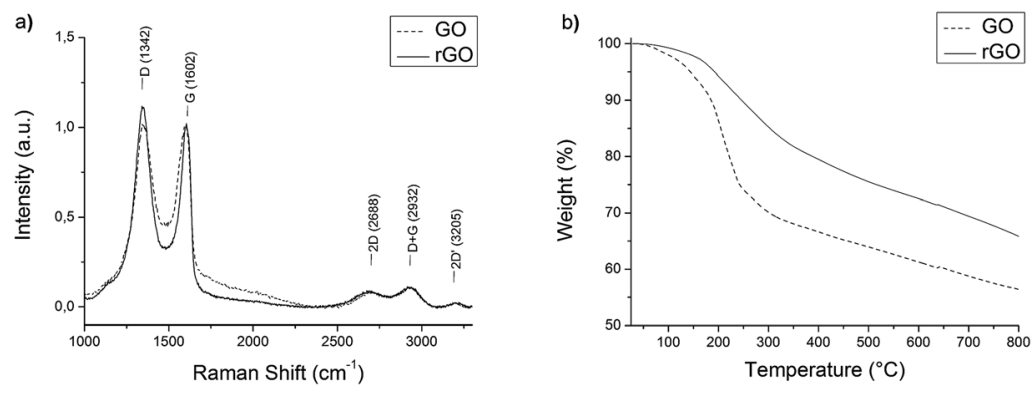

Fig. 3 (a) Raman spectra and (b) thermogravimetric analysis of GO and rGO. 
various concentrations. To ensure realistic conditions, a test gas was mixed with a constant flow of synthetic air. An operating temperature of $85{ }^{\circ} \mathrm{C}$ was chosen to exclude the influence of humidity on the response of the sensor (Fig. SI $8 \dagger$ ). Furthermore, heating to $85{ }^{\circ} \mathrm{C}$ improved the response time $(<1 \mathrm{~min})$, and an increase in the signal change and recovery rate was found. Fig. 4 displays the change of the relative resistance $\left(R / R_{0}\right)$ in the presence of different concentrations of $\mathrm{NO}_{2} . R_{0}$ is hereby the initial resistance right before the addition of a test gas.

The reversibility and concentration dependence of the sensor was evaluated by continuous adsorption and desorption of the different gases. The results for $\mathrm{NO}_{2}$ are shown in Fig. 4, and the changes in conductivity for other gases are listed in Table 1. A linear behaviour within the applied concentration range (25-150 ppm), a sensitivity of $0.56 \mathrm{ppm}^{-1}$ and a detection limit of $0.3 \mathrm{ppm}(S / N=3)$ were observed for $\mathrm{NO}_{2}$. In contrast to the signal drop for $\mathrm{NO}_{2}$, the adsorption of $\mathrm{H}_{2}$ and $\mathrm{CH}_{4}$ led to an increase in electrical conductance. The electron withdrawing effect of the adsorbed $\mathrm{NO}_{2}$ leads to more positive charge carriers since rGO is described to show a p-type semiconducting behaviour. ${ }^{16,38}$ Gases such as $\mathrm{H}_{2}$ or $\mathrm{CH}_{4}$ cannot act as electron donors or acceptors. It can be assumed that previously physisorbed molecules from synthetic air (most probably oxygen) at the surface of the graphene are replaced by $\mathrm{H}_{2}$ or $\mathrm{CH}_{4}$, leading to an increase in electrical resistance..$^{39,40}$

These results clearly demonstrate that rGO on the one hand is an excellent sensor material for detecting gases. In contrast to metal oxide sensors, which are the most common gas sensors so far, sensors based on graphene can be operated at low temperatures with fast response times. On the other hand it is demonstrated that graphene based sensors lack selectivity. Nearly all adsorbates will result in signal changes, therefore they only can be used in detecting analytes in an atmosphere of inert gases. ${ }^{41-48}$ To overcome this drawback graphene itself may be doped by nitrogen, as was theoretically calculated by Ma et al. ${ }^{49}$ Another possibility is to functionalize graphene or to create graphene nanocomposites. ${ }^{50,51}$

Chemical insertion of ODA led to slightly increased signal changes towards $\mathrm{H}_{2}$ and $\mathrm{CH}_{4}$. For $\mathrm{NO}_{2}$ detection almost no influence of the modification could be

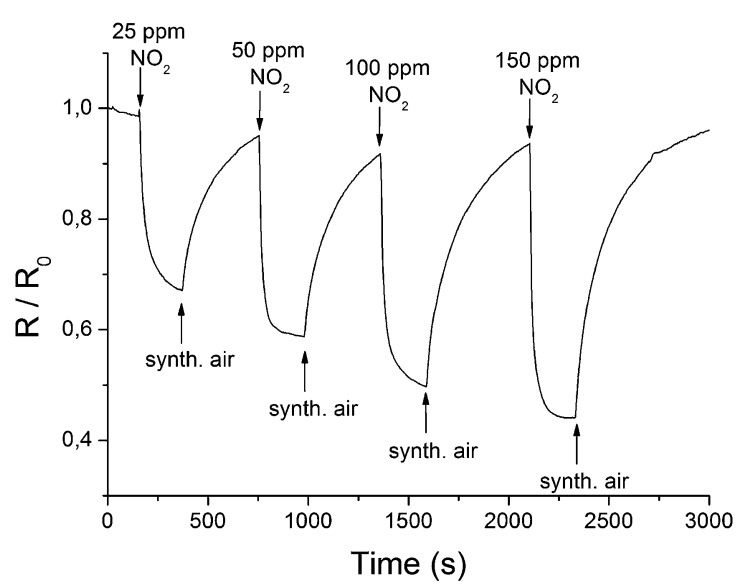

Fig. 4 Changes in the electrical resistance of a $\mathrm{rGO}$ layer at $85^{\circ} \mathrm{C}$ in the presence of different concentrations of $\mathrm{NO}_{2}$. 
Table 1 Comparison of the response of unmodified $\mathrm{rGO}$ and modifications of $\mathrm{rGO}$ in the presence of different concentrations of $\mathrm{NO}_{2}, \mathrm{H}_{2}$ and $\mathrm{CH}_{4}$ at $85{ }^{\circ} \mathrm{C}$

\begin{tabular}{lllllll}
\hline & \multicolumn{2}{l}{ Signal $\left[R / R_{0}\right]$} & & & \\
\cline { 2 - 7 } Gases & rGO & rGO-ODA & rGO-TiO & rGO-MnO & rGO-Pd & rGO-Pt \\
\hline $150 \mathrm{ppm} \mathrm{NO}_{2}$ & 0.419 & 0.436 & 0.718 & 0.929 & 0.358 & 0.758 \\
$125 \mathrm{ppm} \mathrm{NO}_{2}$ & 0.430 & 0.437 & 0.733 & 0.958 & 0.387 & 0.810 \\
$100 \mathrm{ppm} \mathrm{NO}_{2}$ & 0.445 & 0.442 & 0.751 & 0.959 & 0.441 & 0.840 \\
$75 \mathrm{ppm} \mathrm{NO}_{2}$ & 0.494 & 0.456 & 0.768 & 0.969 & 0.461 & 0.849 \\
$50 \mathrm{ppm} \mathrm{NO}_{2}$ & 0.545 & 0.481 & 0.773 & 0.971 & 0.489 & 0.861 \\
$25 \mathrm{ppm} \mathrm{NO}_{2}$ & 0.578 & 0.559 & 0.801 & 0.974 & 0.607 & 0.870 \\
$5000 \mathrm{ppm} \mathrm{H}_{2}$ & 1.035 & 1.037 & 1.026 & 1.017 & 1.615 & 1.135 \\
$3000 \mathrm{ppm} \mathrm{H}_{2}$ & 1.025 & 1.027 & 1.011 & 1.012 & 1.508 & 1.095 \\
$1000 \mathrm{ppm} \mathrm{H}_{2}$ & 1.012 & 1.004 & 1.004 & 1.010 & 1.188 & 1.049 \\
$500 \mathrm{ppm} \mathrm{H}_{2}$ & 1.005 & 1.000 & 1.001 & 1.004 & 1.114 & 1.011 \\
$10000 \mathrm{ppm} \mathrm{CH}_{4}$ & 1.033 & 1.069 & 1.021 & 1.022 & 1.029 & 1.023 \\
$5000 \mathrm{ppm} \mathrm{CH}_{4}$ & 1.023 & 1.048 & 1.012 & 1.019 & 1.019 & 1.016 \\
$3000 \mathrm{ppm} \mathrm{CH}_{4}$ & 1.015 & 1.021 & 1.008 & 1.010 & 1.010 & 1.010 \\
$1000 \mathrm{ppm} \mathrm{CH}_{4}$ & 1.006 & 1.004 & 1.000 & 1.006 & 1.005 & 1.002 \\
\end{tabular}

found. The composite material with $\mathrm{TiO}_{2}$ showed unaffected behaviour towards $\mathrm{H}_{2}$ and $\mathrm{CH}_{4}$, but a lower response towards $\mathrm{NO}_{2}$. This indicates that the adsorption sites for $\mathrm{NO}_{2}$ may be blocked. Electrodes coated with rGO which was doped with $\mathrm{MnO}_{2}$ showed almost no signal change for all of the utilized gases. Therefore, this modification could only be used as a reference signal to compensate for small fluctuations in temperature. The most significant change in response was achieved by chemical doping with Pd- and Pt-nanoparticles. The resulting composite materials showed an increased sensitivity towards $\mathrm{H}_{2}$ (Fig. SI 9b广). The same observations with comparable results have been made with carbon nanotubes decorated with $\mathrm{Pt}$ and multilayered graphene nanoribbons doped with $\mathrm{Pd}{ }^{\mathbf{2 4 , 5 2}}$ Though the composite material with rGO is easier to produce and process.

In summary, all of the composites of rGO presented here are simple to prepare and show different behaviour in their electrical conductance in the presence of various gases. The sensor responses of different functionalized rGO materials towards $\mathrm{NO}_{2}, \mathrm{H}_{2}$ and $\mathrm{CH}_{4}$ (Fig. 5) are summarized in Table 1.

\subsection{Principal component analysis for pattern recognition of different gases}

The altered sensitivity of every sensor towards different analyte gases enables pattern recognition using multivariate analysis based on principal component analysis (PCA). With this chemometric technique it is possible to simplify multidimensional datasets without crucial loss of information. Here the data matrix (Table 1) contains the normalized sensor response of each sensor material to a certain gas and concentration. This multidimensional matrix can be reduced to two principle components (PC1, PC2). The variance of PC1 (95.75\%) and PC2 $(2.94 \%)$ is above $98 \%$ and therefore these components already contain significant information to represent the data in two dimensions (Fig. 6). Clear separation between the clusters representing individual gases with no overlap and a 
a)

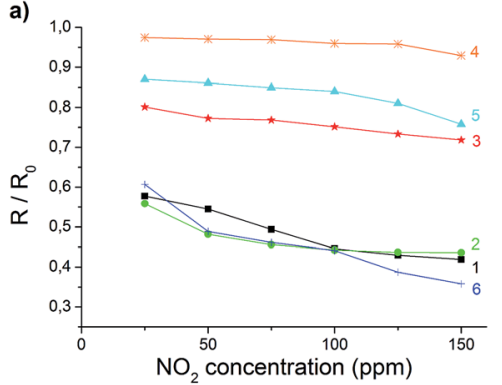

b)

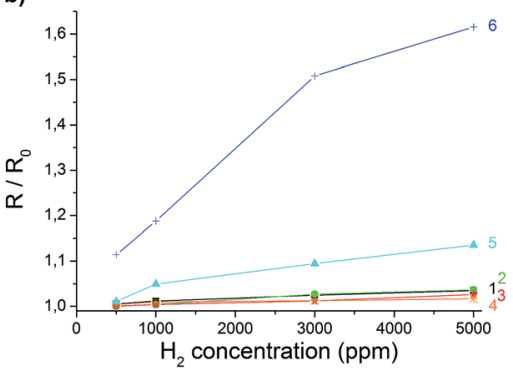

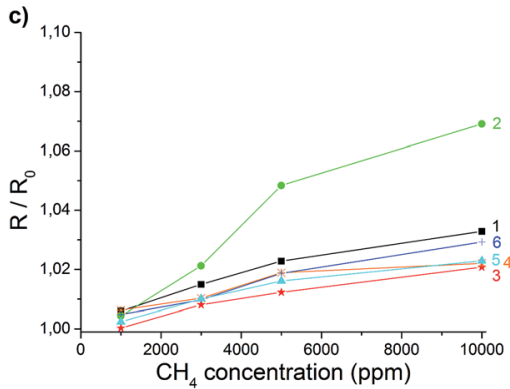

Fig. 5 Changes in electrical resistance of $\mathrm{rGO}(1)$ and graphene composites (2 to 6) for different concentrations of $\mathrm{NO}_{2}(\mathrm{a}), \mathrm{H}_{2}$ (b) and $\mathrm{CH}_{4}$ (c). The composites are $\mathrm{rGO}-\mathrm{ODA}$ (2), $\mathrm{rGO}-\mathrm{TiO}_{2}$ (3), rGO- $\mathrm{MnO}_{2}$ (4), rGO-Pt (5), and $\mathrm{rGO}-\mathrm{Pd}(6)$.

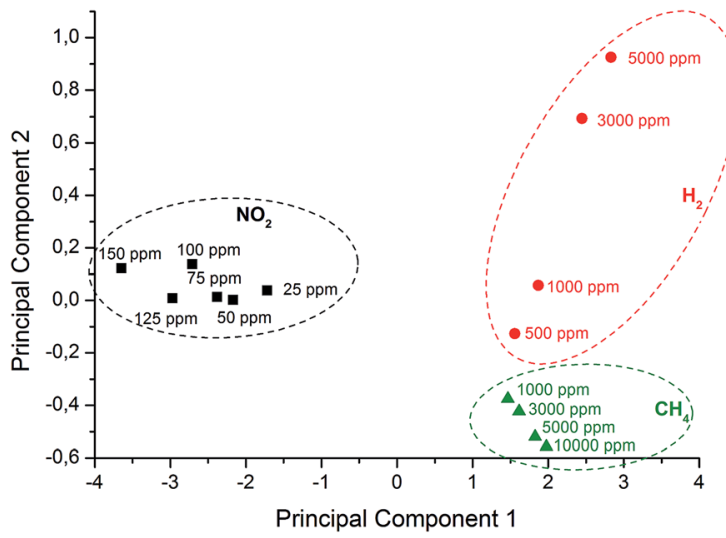

Fig. 6 Pattern analysis based on PCA using six different sensors (modified with $\mathrm{rGO}, \mathrm{rGO}-$ ODA, rGO- $\mathrm{TiO}_{2}, \mathrm{rGO}-\mathrm{MnO}_{2}, \mathrm{rGO}-\mathrm{Pd}$, and $\mathrm{rGO}-\mathrm{Pt}$ ) for various concentrations of individual gases $\left(\mathrm{NO}_{2}, \mathrm{H}_{2}\right.$, and $\left.\mathrm{CH}_{4}\right)$.

recognizable trend of concentration allows the identification of all analytes with a set of six different electrodes.

Regeneration of the sensor material still has to be further investigated. In this study the regeneration was mostly performed by a short term heat treatment at 
$230{ }^{\circ} \mathrm{C}$ between measurements. Furthermore this step ensures the complete desorption of any physisorbed impurities as demonstrated by the TGA measurements. In an application this could be realized by different operation temperatures with short regeneration cycles.

\section{Conclusion}

Reduced graphene oxide is an ideal sensor material for chemiresistive gas sensors due to its simple preparation and functionalization enabling altered sensitivities. Compared to commercial solid state gas sensors, rGO sensors have the advantage of operating at the low temperature of $85{ }^{\circ} \mathrm{C}$ to exclude the strong influences of humidity. It was demonstrated that the spin coating of rGO composites results in reproducible sensor behaviour suitable for well established fabrication technologies. In a chemiresistor setup, unmodified rGO showed high sensitivity towards $\mathrm{NO}_{2}$ on the ppm level at ambient conditions. The sensor was rather unselective and also showed responses towards $\mathrm{H}_{2}$ and $\mathrm{CH}_{4}$. Upon different functionalizations it was possible to achieve different sensor behaviors for different gases. This can be used to apply PCA to discriminate between each individual gas. It is expected that this approach could be extended to build up sensor arrays for detecting the concentration of many individual gases in a complex matrix at low temperatures.

\section{Acknowledgements}

This work has been supported by Infineon Technologies AG, Regensburg. The authors thank Stefan Wilhelm and Christoph Fenzl for TEM studies and Gerhard Poeppel for the help with PCA.

\section{References}

1 J. M. Samet, Inhalation Toxicol., 2007, 19, 1021-1027.

2 P. T. Moseley, Meas. Sci. Technol., 1997, 8, 223-237.

3 G. Korotcenkov, Mater. Sci. Eng., B, 2007, 139, 1-23.

4 A. Tricoli, M. Righettoni and A. Teleki, Angew. Chem., Int. Ed., 2010, 49, 76327659.

5 P. Bondavalli, P. Legagneux and D. Pribat, Sens. Actuators, B, 2009, 140, 304318.

6 S. Mao, G. Lu and J. Chen, J. Mater. Chem. A, 2014, 2, 5573-5579.

7 F. Schedin, A. K. Geim, S. V. Morozov, E. W. Hill, P. Blake, M. I. Katsnelson and K. S. Novoselov, Nat. Mater., 2007, 6, 652-655.

8 W. Yuan and G. Shi, J. Mater. Chem. A, 2013, 1, 10078-10091.

9 S. Basu and P. Bhattacharyya, Sens. Actuators, B, 2012, 173, 1-21.

10 Q. He, S. Wu, Z. Yin and H. Zhang, Chem. Sci., 2012, 3, 1764-1772.

11 A. K. Geim and K. S. Novoselov, Nat. Mater., 2007, 6, 183-191.

12 C. Soldano, A. Mahmood and E. Dujardin, Carbon, 2010, 48, 2127-2150.

13 W. S. Hummers Jr and R. E. Offeman, J. Am. Chem. Soc., 1958, 80, 1339-1339.

14 D. Li, M. B. Müller, S. Gilje, R. B. Kaner and G. G. Wallace, Nat. Nanotechnol., 2008, 3, 101-105. 
15 C. Gómez-Navarro, R. T. Weitz, A. M. Bittner, M. Scolari, A. Mews, M. Burghard and K. Kern, Nano Lett., 2007, 7, 3499-3503.

16 S. Gilje, S. Han, M. Wang, K. L. Wang and R. B. Kaner, Nano Lett., 2007, 7, 3394-3398.

17 J. T. Robinson, F. K. Perkins, E. S. Snow, Z. Wei and P. E. Sheehan, Nano Lett., 2008, 8, 3137-3140.

18 Y.-H. Zhang, Y.-B. Chen, K.-G. Zhou, C.-H. Liu, J. Zeng, H.-L. Zhang and Y. Peng, Nanotechnology, 2009, 20, 185504.

19 G. Eda, G. Fanchini and M. Chhowalla, Nat. Nanotechnol., 2008, 3, 270-274.

20 Y. Xu and G. Shi, J. Mater. Chem., 2011, 21, 3311-3323.

21 O. Leenaerts, B. Partoens and F. M. Peeters, Phys. Rev. B: Condens. Matter Mater. Phys., 2008, 77, 125416.

22 S. Mao, S. Cui, G. Lu, K. Yu, Z. Wen and J. Chen, J. Mater. Chem., 2012, 22, 11009-11013.

23 Q. Huang, D. Zeng, H. Li and C. Xie, Nanoscale, 2012, 4, 5651-5658.

24 J. L. Johnson, A. Behnam, S. J. Pearton and A. Ural, Adv. Mater., 2010, 22, 48774880.

25 S. Wang, P.-J. Chia, L.-L. Chua, L.-H. Zhao, R.-Q. Png, S. Sivaramakrishnan, M. Zhou, R. G.-S. Goh, R. H. Friend, A. T.-S. Wee and P. K.-H. Ho, Adv. Mater., 2008, 20, 3440-3446.

26 S. Chen, J. Zhu, X. Wu, Q. Han and X. Wang, ACS Nano, 2010, 4, 2822-2830.

27 X. Li, J. Niu, J. Zhang, H. Li and Z. Liu, J. Phys. Chem. B, 2003, 107, 2453-2458.

28 C. Xu, X. Wang and J. Zhu, J. Phys. Chem. C, 2008, 112, 19841-19845.

29 S. Bae, H. Kim, Y. Lee, X. Xu, J.-S. Park, Y. Zheng, J. Balakrishnan, T. Lei, H. Ri Kim, Y. I. Song, Y.-J. Kim, K. S. Kim, B. Özyilmaz, J.-H. Ahn, B. H. Hong and S. Iijima, Nat. Nanotechnol., 2010, 5, 574-578.

30 K. S. Kim, Y. Zhao, H. Jang, S. Y. Lee, J. M. Kim, K. S. Kim, J.-H. Ahn, P. Kim, J.-Y. Choi and B. H. Hong, Nature, 2009, 457, 706-710.

31 S. Stankovich, D. A. Dikin, R. D. Piner, K. A. Kohlhaas, A. Kleinhammes, Y. Jia, Y. Wu, S. T. Nguyen and R. S. Ruoff, Carbon, 2007, 45, 1558-1565.

32 M. A. Pimenta, G. Dresselhaus, M. S. Dresselhaus, L. G. Cançado, A. Jorio and R. Saito, Phys. Chem. Chem. Phys., 2007, 9, 1276.

33 I. Jung, D. A. Field, N. J. Clark, Y. Zhu, D. Yang, R. D. Piner, S. Stankovich, D. A. Dikin, H. Geisler, C. A. Ventrice and R. S. Ruoff, J. Phys. Chem. C, 2009, 113, 18480-18486.

34 I. Jung, D. A. Dikin, R. D. Piner and R. S. Ruoff, Nano Lett., 2008, 8, 4283-4287.

35 J. Wöllenstein, M. Burgmair, G. Plescher, T. Sulima, J. Hildenbrand, H. Böttner and I. Eisele, Sens. Actuators, B, 2003, 93, 442-448.

36 K. P. Loh, Q. Bao, P. K. Ang and J. Yang, J. Mater. Chem., 2010, 20, 2277-2289.

37 S. Bai and X. Shen, RSC Adv., 2012, 2, 64-98.

38 J. D. Fowler, M. J. Allen, V. C. Tung, Y. Yang, R. B. Kaner and B. H. Weiller, ACS Nano, 2009, 3, 301-306.

39 M. Gautam and A. H. Jayatissa, Mater. Sci. Eng., C, 2011, 31, 1405-1411.

40 Y. Dan, Y. Lu, N. J. Kybert, Z. Luo and A. T. C. Johnson, Nano Lett., 2009, 9, 1472-1475.

41 H. Y. Jeong, D.-S. Lee, H. K. Choi, D. H. Lee, J.-E. Kim, J. Y. Lee, W. J. Lee, S. O. Kim and S.-Y. Choi, Appl. Phys. Lett., 2010, 96, 213105.

42 J. L. Johnson, A. Behnam, S. J. Pearton and A. Ural, Adv. Mater., 2010, 22, 48774880. 
43 J. Zou, L. J. Hubble, K. S. Iyer and C. L. Raston, Sens. Actuators, B, 2010, 150, 291-295.

44 B. H. Chu, C. F. Lo, J. Nicolosi, C. Y. Chang, V. Chen, W. Strupinski, S. J. Pearton and F. Ren, Sens. Actuators, B, 2011, 157, 500-503.

45 W. Li, X. Geng, Y. Guo, J. Rong, Y. Gong, L. Wu, X. Zhang, P. Li, J. Xu, G. Cheng, M. Sun and L. Liu, ACS Nano, 2011, 5, 6955-6961.

46 V. Tjoa, W. Jun, V. Dravid, S. Mhaisalkar and N. Mathews, J. Mater. Chem., 2011, 21, 15593-15599.

47 W. Yuan, A. Liu, L. Huang, C. Li and G. Shi, Adv. Mater., 2013, 25, 766-771.

48 F. Zhang, F. M. Yasin, X. Chen, J. Mo, C. L. Raston and H. Zhang, RSC Adv., 2013, 3, 25166-25174.

49 C. Ma, X. Shao and D. Cao, Sci. China: Chem., 2014, 57, 911-917.

50 Y. Yang, C. Tian, J. Wang, L. Sun, K. Shi, W. Zhou and H. Fu, Nanoscale, 2014, 6, 7369-7378.

51 L. Huang, Z. Wang, J. Zhang, J. Pu, Y. Lin, S. Xu, L. Shen, Q. Chen and W. Shi, ACS Appl. Mater. Interfaces, 2014, 6, 7426-7433.

52 A. Kaniyoor, R. I. Jafri, T. Arockiadoss and S. Ramaprabhu, Nanoscale, 2009, 1, 382-386. 